East African Medical Journal Vol. 79 No. 3 March 2002

BOWEL FUNCTION FOLLOWING PRIMARY REPAIR OF ANORECTAL MALFORMATIONS AT KENYATTA NATIONAL HOSPITAL

C. N. Kigo, MBChB, MMed (Surg), District Surgeon, Murang'a District Hospital, P.O. Box 625, Murang'a, Kenya and J.M. Ndung'u, MBChB, MMed (Surg),

Senior Lecturer, Department of Surgery, College of Health Sciences, University of Nairobi, P.O. Box 19676, Nairobi, Kenya.

Request for reprints to: Dr. C.N. Kigo, Murang'a District Hospital, P.O. Box 625, Murang'a, Kenya

\title{
BOWEL FUNCTION FOLLOWING PRIMARY REPAIR OF ANORECTAL MALFORMATIONS AT KENY ATTA NATIONAL HOSPITAL
}

\author{
C. N. KIGO and J.M. NDUNG'U
}

\begin{abstract}
Objectives: To evaluate bowel function following primary repair of anorectal malformation. Design: A ten-year retrospective study.

Setting: Kenyatta National Hospital, Nairobi, Kenya.

Subjects: All patients with anorectal malformations attended to at Kenyatta National Hospital (KNH) within the study period who had posterior sagittal repair as a primary definitive procedure. All the children were over three years of age, toilet trained, and had their colostomies closed with an adaptation period of at least six months.

Results: Posterior sagittal repair was used to repair anorectal malformations in 352 patients. One hundred and ninety three patients were evaluated. Overall voluntary bowel movement (VBM) was achieved in $71.5 \%$ of the patients, soiling was present in $21.2 \%$ of the patients and constipation in $7.3 \%$ of the patients. More than seventy nine per cent of children who had their colostomy fashioned before the age of one month achieved VBM, while $61.1 \%$ of the patients achieved VBM when the colostomy was fashioned after five years. Overall, $77.0 \%$ of the females achieved VBM compared to $63.8 \%$ of males. Patients with a perineal fistula achieved VBM in $79.1 \%$ of males and $75.0 \%$ of females, $76.0 \%$ with vestibular fistula, $73.9 \%$ with a recto-urethral fistula, $56.0 \%$ of anorectal anomalies without a fistula, $25.0 \%$ of vaginal fistulae and $12.5 \%$ in vesical fistulae. Overall patients with sacral defects achieved VBM in $25.9 \%$ compared to $78.9 \%$ in patients with a normal sacrum. The patients with low anomalies achieved VBM in $75.4 \%$ compared to $46.1 \%$ with high anomalies.

Conclusions: Posterior sagittal repair has been used to repair all anorectal malformations and has improved the quality of life of our patients, with better functional results expected in female patients, early colostomy fashioning and definitive repair, low or simple anomalies, and absence of sacral defects. The repair was associated with low morbidity and mortality.
\end{abstract}

\section{INTRODUCTION}

Posterior sagittal repair has been the definitive management procedure for children with anorectal malformations (ARM) since 1987 in KNH. Since then posterior sagittal repair has been adapted exclusively as the procedure of choice for the correction of the entire spectrum of ARMs. It has provided a new hope for the children with this anomaly(1). Faecal continence is the primary goal following repair. Control of bowel function previously provided the greatest challenge $(1,2)$. The proper care of newborns' anorectal anomalies consists of: proper decision making with or without the creation of a colostomy in the neonatal period, perfect execution of the subsequent definitive procedure, and knowledge of the long-term anticipated complications.

The functional results depend on the location of the associated fistula, age of the patient at definitive surgery, type of sacrum, and the length of time before fashioning the colostomy. Faecal incontinence is a serious problem that provokes social segregation and psychological sequelae. Post-operatively, at least $25-30 \%$ will suffer from faecal incontinence(3). In addition another $30 \%$ will suffer from other functional defaecation disorders such as constipation, occasional soiling, and faecal incontinence during periods of diarrhoea $(3,4)$.

\section{MATERIALS AND METHODS}

This was a descriptive retrospective study over a period of ten years from 1st January 1990 to 31 st December 1999. Information about bowel function in anorectal anomalies was collected by the use of a pretested questionnaire. The case records of all the patients with ARM treated within the study period were retrieved from the department of records of $\mathrm{KNH}$. Then the information obtained in these records including details of clinical examination, radiological and operative findings were reviewed to determine the structural abnormalities, complications and the outcome in these patients.

All the patients with anorectal anomalies were grouped according to Pena's classification(3). Continence was graded according to signs and symptoms recorded on the first visit six 
months after closure of colostomy. Only the patients who were three years of age and toilet trained were evaluated. The patients with inability to control defaecation voluntarily were considered incontinent, and those with various degrees of soiling including those with associated constipation were also considered incontinent.

After the collection exercise, coding was done for all the questionnaires. Data entry was then done into SPSS data sheet. Analysis of the data was done using SPSS V.8 computer programme.

\section{RESULTS}

The study was carried out between 1st January 1990 and 31 st December 1999 in KNH. Posterior sagittal repair was carried out on 352 patients, 193 of these were evaluated while 149 patients were excluded (Table 1).

Characteristics of the patients evaluated: Males were $80(43.0 \%)$ and females $113(57.0 \%)$. Fourteen per cent of the patients had sacral abnormalities, $86.5 \%$ had low anomalies, $92.8 \%$ had pre-op colostomy ( $87.5 \%$ of males and $95.6 \%$ of females). A divided sigmoid colostomy was fashioned in $75.8 \%$ and a transverse colostomy in $24.2 \%$ of the patients. The majority of the patients (90.5\%) wait for longer than six months for definitive surgery after colostomy fashioning.
Table 1

\begin{tabular}{lll} 
& \multicolumn{2}{c}{ Exclusion criteria } \\
\hline & & Frequency \\
\hline Evaluated & & 193 \\
Excluded & $<6$ months post-op & 23 \\
& Lost to follow-up & 52 \\
& Colostomy still open & 27 \\
& Files missing & 45 \\
& Mortality & 2 \\
\hline \multirow{2}{*}{ Total excluded } & & 149 \\
\hline
\end{tabular}

Table 2

Bowel function in primury definitive surgery

\begin{tabular}{lrr}
\hline & Frequency & Valid \% \\
\hline VBM & 138 & 71.5 \\
Soiling grade 1 & 19 & 9.8 \\
Soiling grade 2 & 15 & 7.3 \\
Soiling grade 3 & 8 & 4.1 \\
Constipation grade 1 & 4 & 2.1 \\
Constipation grade 2 & 3 & 1.6 \\
Constipation grade 3 & 7 & 3.6 \\
\hline & 193 & 100.0 \\
\hline
\end{tabular}

Table 3

Bowel function by the age at colostomy fashioning

\begin{tabular}{|c|c|c|c|c|c|c|}
\hline \multirow{3}{*}{$\begin{array}{l}\text { Age at } \\
\text { colostomy } \\
\text { fashioning }\end{array}$} & \multirow{3}{*}{$\begin{array}{l}\text { No. of } \\
\text { cases }\end{array}$} & \multirow{3}{*}{ VBM } & \multicolumn{3}{|c|}{ Bowel function } & \\
\hline & & & & & \multicolumn{2}{|c|}{ Constipation } \\
\hline & & & Grade 1 & Grade $>1$ & Grade 1 & Grade $>1$ \\
\hline $0-1$ month & 104 & $79(76.0)$ & $7(6.7)$ & $12(11.5)$ & $4(3.8)$ & $2(1.9)$ \\
\hline $2-6$ months & 50 & $37(74.0)$ & $4(8.0)$ & $3(6.0)$ & $6(12.0)$ & 0 \\
\hline 7.12 months & 6 & $4(66.9)$ & $1(6.7)$ & $1(16.7)$ & 0 & 0 \\
\hline$>12$ months & 18 & $11(61.1)$ & $4(22.2)$ & $3(16.7)$ & 0 & 0 \\
\hline Total & 178 & $131(73.5)$ & $18(10.1)$ & $18(10.8)$ & $10(5.6)$ & $2(1,1)$ \\
\hline
\end{tabular}

Table 4

\begin{tabular}{|c|c|c|c|c|c|c|}
\hline \multicolumn{7}{|c|}{ Bowel function in males } \\
\hline \multirow[t]{2}{*}{ Males anomalies } & \multirow[t]{2}{*}{$\begin{array}{l}\text { No. of } \\
\text { cases }\end{array}$} & \multirow[t]{2}{*}{ VBM } & \multicolumn{2}{|c|}{$\begin{array}{l}\text { Bowel function } \\
\text { Soiling }\end{array}$} & \multicolumn{2}{|c|}{ Constipation } \\
\hline & & & Grade 1 & Grade $>1$ & Grade 1 & Grade $>1$ \\
\hline Perineal fistula & 24 & $19(79.1)$ & 0 & 0 & $3(12.4)$ & $3(3.8)$ \\
\hline Rectourethral fistula & 23 & $17(73.9)$ & $6(20.1)$ & $1(4.3)$ & 0 & 0 \\
\hline Rectovesical fistula & 8 & $1(12.5)$ & 0 & $7(87.5)$ & 0 & 0 \\
\hline ARM without a fistula & 25 & $14(56.0)$ & $8(32.0)$ & $3(12.0)$ & 0 & 0 \\
\hline Total & 80 & $51(63.8)$ & $14(5.0)$ & $11(13.8)$ & $3(3.8)$ & $3(3.8)$ \\
\hline
\end{tabular}


Table 5

Bowel function in females

\begin{tabular}{|c|c|c|c|c|c|c|}
\hline \multirow[t]{2}{*}{ Female anomalies } & \multirow[t]{2}{*}{$\begin{array}{r}\text { No. of } \\
\text { cases }\end{array}$} & \multirow{2}{*}{$\mathrm{VBM}$} & \multicolumn{2}{|c|}{ Bowel function } & \multicolumn{2}{|c|}{ Constipation } \\
\hline & & & Grade 1 & Grade $>1$ & Grade 1 & Grade $>1$ \\
\hline Perineal fistula & 8 & $6(75.0)$ & $1(12.5)$ & 0 & 0 & $1(12.5)$ \\
\hline Vestibular fistula & 96 & $73(76.0)$ & $11(11.5)$ & $8(8.3)$ & $1(1.0)$ & $3(3.1)$ \\
\hline Vaginal fistula & 8 & $2(25.0)$ & $2(25.0)$ & $3(37.5)$ & 0 & $1(12.5)$ \\
\hline Persistent cloaca & & & & & & \\
\hline$<3 \mathrm{~cm}$ channel & 1 & 0 & $1(100)$ & 0 & 0 & 0 \\
\hline Total & 113 & $87(77.0)$ & $15(13.3)$ & $11(9.7)$ & $1(0.8)$ & $5(4.4)$ \\
\hline
\end{tabular}

Table 6

Bowel function by age at definitive surgery

\begin{tabular}{|c|c|c|c|c|c|c|}
\hline \multirow{2}{*}{$\begin{array}{l}\text { Age at } \\
\text { definitive } \\
\text { surgery }\end{array}$} & \multirow[t]{2}{*}{$\begin{array}{l}\text { No. of } \\
\text { cases }\end{array}$} & \multirow[t]{2}{*}{ VBM } & \multicolumn{2}{|c|}{$\begin{array}{l}\text { Bowel function } \\
\text { Soiling }\end{array}$} & \multicolumn{2}{|c|}{ Constipation } \\
\hline & & & Grade 1 & Grade $>1$ & Grade 1 & Grade $>1$ \\
\hline $0-6$ months & 21 & $17(81.1)$ & 0 & 0 & 0 & $4(19.0)$ \\
\hline $7-12$ months & 31 & $24(77.4)$ & 0 & $1(3.2)$ & $4(12.9)$ & $2(6.4)$ \\
\hline 13-24 months & 78 & $54(69.2)$ & $8(10.2)$ & $14(17.9)$ & 0 & $2(1.3)$ \\
\hline $25-60$ months & 50 & $35(70.0)$ & $8(16.0)$ & $5(10.0)$ & 0 & $2(4.0)$ \\
\hline$>60$ months & 13 & $8(61.5)$ & $3(23.0)$ & $2(15.4)$ & 0 & 0 \\
\hline Total & 193 & $138(71.5)$ & $19(9.8)$ & $22(11.4)$ & $4(2.1)$ & $10(5.2)$ \\
\hline
\end{tabular}

Table 7

Bowel function by the type of sacrum

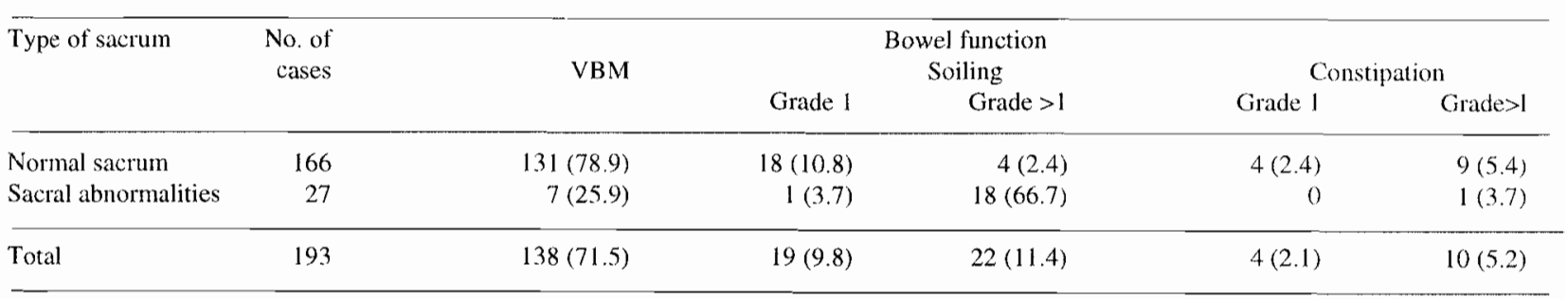

By the age of five years the majority of patients (93.3\%) have had definitive surgery. Only $10.9 \%$ had definitive surgery by the age of six months. Of the 80 male patients studied, $25(31.2 \%)$ had anorectal malformations without a fistula, $24(30.0 \%)$ had a perineal fistula, $23(28.8 \%)$ had a rectourethral fistula and eight $(10.0 \%)$ had a rectovesical fistula. There was no patient with rectal atresia. Of the 113 female patients studied, 95 (84.1\%) had a vestibular fistula, eight $(7.1 \%)$ had a perineal fistula, another eight $(7.1 \%)$ a vaginal fistula and two $(1.7 \%)$ had a persistent cloaca with a common channel of less than $3 \mathrm{~cm}$.

\section{DISCUSSION}

Anorectal malformations are a common problem in Kenya. In Kenyatta National Hospital (KNH), the main referral hospital, approximately 35 patients undergo definitive repair every year currently up from 23 in 1995 (1,2). A good repair saves a child so unfortunate to be born with this anomaly a lifetime of misery and social seclusion. We evaluated children over three years old, toilet trained and six months after closure of colostomy.

Voluntary bowel movement (VBM) is the most valuable sign of faecal control. Overall in this series $71.5 \%$ of all the patients achieved VBM. Also noted is the high proportion of patients with low anomalies and normal sacra(1). Most series report a VBM of $75 \%(3,4)$. Overall soiling was present in $21.2 \%$ of all the patients (Table 2 ) . Historical data show that approximately $25 \%$ of patients suffer some form of faecal incontinence $(3,4)$. Faecal continence is dependent on intact sensation, lack of scarring, normal peristalsis, intact neural pathways, psychological factors and anatomical factors (functioning internal and external sphincters). In this series constipation was present in $7.3 \%$ of the patients and was not a serious problem despite a high incidence of low anomalies( 1 ). Most series report a high incidence of constipation as a complication of internal anal sphincter saving procedures in repair of simple or low anomalies, and dysganglionosis of distal rectal pouch $(5,6)$.

Divided sigmoid colostomy is the standard colostomy 
fashioned prior to definitive surgery in $\mathrm{KNH}$. This type of colostomy has a low incidence of prolapse, is completely diverting, and minimises urinary tract contamination in patients with rectourinary fistulae and vesico-ureteric reflux $(1,7,8)$. All the patients evaluated with transverse loop colostomy had this type of colostomy fashioned in peripheral hospitals prior to referral for definitive surgery at KNH. Transverse loop colostomy is not totally diverting and allows some stools to reach the distal stoma $(7,8)$. Overall, the children who had colostomy fashioned before the age of one month had better bowel control (VBM of $79.1 \%$ ) compared to those fashioned thereafter (VBM was $61.1 \%$ after one year). Early colostomy reduces the incidence of rectal ectasia developing before the main repair(9). The majority of our patients $(90.5 \%)$ wait longer than six months for definitive surgery after colostomy fashioning. This was due to the rising numbers requiring definitive surgery. In this series, the duration between colostomy fashioning and definitive surgery does not influence the outcome of bowel function. Theoretically, the patients who wait for shorter duration should have better results, since the parents arc cooperative and they are not exposed to the traumatising colostomy for a long time, anal dilations are easier and the patients do not have memories of painful manoeuvres(7).

The type of sacrum was evaluated by a review of radiographs, sacral ratio(3) was measured (sacral ratio $>0.74$ was considered normal). In this series a normal sacrum is associated with good prognosis for bowel control. About eighty per cent of patients with a normal sacrum (166 patients) achieved VBM. Those with sacral abnormalities (27 patients) achieved VBM in only $25.9 \%$ with $66.7 \%$ having severe soiling. The spectrum of sacral anomalies found included fused and hemisacrum.

Patients who had primary definitive repair before the age of one month (21 patients) achicved VBM in $81.1 \%$ while those operated after five years (13 patients), only $61.5 \%$ of the patients achicved VBM. Patients operated before the age of six months achieved better results, as the rectum is placed in the right position early in life restoring the anorectal continuity, encouraging new synapses to develop early providing better sensation and better function of sphincteric mechanism $(7,10,11,12)$.

Females had better functional results than males(13). In the current series, $63.8 \%$ of males and $77.0 \%$ of females achieved VBM. Males had a higher incidence of high anomalies and sacral defects. Overall $16.6 \%$ of the patients had a perineal fistula and achicved VBM in $79.1 \%$ of males and $75.0 \%$ in females. Anorectal malformations without a fistula are the commonest malformation in males with $56 \%$ achieving VBM. Five patients with Downs' syndrome had anorectal malformations and all of these had no fistula. Rectourethral fistula is the third commonest malformation in males (23 patients) and $73.9 \%$ of these patients achieved VBM. Rectovestibular fistula is the commonest anomaly in females (96 patients). Seventy six per cent of these patients achieved VBM. Eight patients with a rectovesical fistula had the worst prognosis for bowel control with only $12.5 \%$ achieving VBM $(87.5 \%$ of these patients had severe soiling).

Re-operation was necessary in $24(12.4 \%)$ patients. Indications for re-operation included severe faecal incontinence and severe constipation. This rate is lower than that previously recorded at $\mathrm{KNH}$ of $15.2 \%$ (2). The mortality has been declining steadily. In the study period there were two $(1.0 \%)$ deaths, the cause of the deaths was severe peritonitis secondary to anastomotic leak after colostomy closure. Previous recorded mortality rate was 29.5\% in 1982 and five per cent in 1995(2).

In conclusion, posterior sagittal repair is a useful way to repair all types of anorectal malformations, with a totally diverting sigmoid colostomy. There is accurate placement of the rectum within the muscle complex under direct vision of the pelvic anatomy. Pena's protocol for assessment(3) of bowel function has been used in this study, making it easier to compare malformations with similar potential for bowel control. On the whole, at KNH one can expect $72 \%$ of children with anorectal malformations and subjected to posterior sagittal repair, to achieve VBM. Good results of bowel control are associated with early age of fashioning the colostomy and early age of definitive surgery. Female sex, absence of sacral defects and low or simple anomalies are also favourable factors.

\section{ACKNOWLEDGEMENTS}

To the Kenyatta National Hospital Ethical and Research Committee for permission to publish the results of the study. We are also indebted to Dr. G.C.N Anangwe for reviewing the manuscript.

\section{REFERENCES}

1. Kigo C. N. Posterior sagittal repair of anorectal malformations in Kenyatta National Hospital. M. Med. Surgery Thesis, University of Nairobi, 2001.

2. Okumu W.J. Pattern of occurrence of anorectal malformations at Kenyatta National Hospital. M. Med. Surgery Thesis. University of Nairobi, 1995.

3. Pena A. Anorectal malformations. Sem. Paediat. Surg. 1995; 4:35-47.

4. Paidas C. N. Faecal incontinence in children with anorectal malformation. Sem. Paediat. Surg. 1997; 6:228-234.

5. Shono T., Nagasaki A. and Hirose R. et al. Acetyl cholinesterase (ACE) staining shows the abnormal innervation of a pulled through rectum in a case of repaired anorectal malformation. Euro. J. Paediat. Surg. 1997; 7:248-51

6. Holschneider A. M., Ure B. M. and Pfromer W. e't al. Innervation patterns of rectal pouch and fistula in anorectal malformation: a preliminaty report. J. Paediat. Surg. 1996: 31:357-62.

7. Shual D. B. and Harrison E. A. Classification of anorectal malformationsInitial approach, diagnostic tests, and colostomy. Sem. Patdiat. Surg. 1997; 6:187 195.

8. Pena A. Preface: Advances in anorectal malformations. Sem. Pediat. Surg. 1997; 6:165-69.

9. Zia-ul-Miraj M. and Brereton R. J. Rectal ectasia associated with anorectal anomalies. J. Paedial. Surg. 1997; 32:621-3.

10. Moore T. C. Advantages of performing the posterior sagittal anoplasty operation at birth. J. Paediat. Surg. 1990; 25:276-77.

11. Frecman N. V. High anorectal anomalies treated by early neonatal operation. J. Paediat. Surg. 1986; 21 : 218-220.

12. Freeman N. V. Anal evoked potential. Z. Kinderchir. 1980; 31: 22-30

13. Mouder W., de Jong E. and Wauters. et al Posterior Sagittal Anorectoplasty functional results of primary and secondary operations in comparison to pull through methods in anorectal malformations. Euro. J. Paediat. Surg. 1995: 5: 170-3.

14. Heinen F. L. The surgical treatment of low anal defects and vestibulat fistulas. Sem. Paediat. Surg. 1997; 6:204-215. 